\title{
The flower of seduction
}

\author{
Hundreds of orchid species lure their pollinators with the \\ promise of sex, only to send them away unfulfilled. Heidi Ledford \\ looks at how dishonesty gives them the evolutionary edge.
} e's a loner and, truth be told, he's

$\mathrm{H}$ never been with a lady before. She's an experienced siren with an irresistible scent. Lightning quick, he zigzags close, strokes her hair and mounts her. But it is only as he engages in a few confused attempts at copulation that the truth dawns: his chosen is not a lady after all. She's a flower.

So goes the tale of the male solitary bee (Andrena nigroaenea) and his seducer, the green and dainty early spider orchid (Ophrys sphegodes). These orchids bloom in the spring, when female bees are still in their nests and the males are young, inexperienced, and likely to encounter an orchid before they find a real mate. During his attempts to mate with the elaborate flower, the bee may pick up a large packet of sticky pollen on his head, ready to deliver to another plant. That's a boon to the orchid; the bee, on the other hand, gets nothing but hot and bothered.

Some 30,000 species strong, orchids are one of the largest groups of flowering plants; collectors covet their elaborate and sometimes rude-looking flowers. But the plants are also unusual in

"To be deceptive means
that the orchids have
less sex, but the sex is
better because it's not
with a close relative."
- Salvatore Cozzolino

"To be deceptive means that the orchids have less sex, but the sex is that some deceptive orchids use to lure insects. Because each species manufactures a unique cocktail, and typically attracts only one species of pollinator, the odours provide a way for biologists to identify species in this class of orchid and to study how they have diversified. These studies may eventually provide evidence for sympatric speciation, the concept that a new species can evolve without being geographically isolated. Indeed, orchids may be one of the most promising ways in which to find evidence for this controversial idea.

At first glance, dishonesty seems to be a bad policy for orchids. Non-rewarding flowers have low pollination rates, and produce only half as much fruit as rewarding flowers ${ }^{1}$. Add nectar to a non-rewarding plant and pollination rates shoot up. If reproductive success was meas-
ured by seed production alone, then deceptive orchids would have lost the evolutionary race long ago. But deception may be worthwhile because it prevents inbreeding and so boosts the quality, rather than quantity, of seeds. Deceived pollinators are more likely to go off in a huff after realizing they've been tricked: they'll take their their dishonesty. Although most insect-pollinated plants pay their pollinators in energyrich nectar, about a third of orchids offer no rewards. Roughly 10,000 species deceive their pollinators by mimicking plants that do provide nectar. And, unique among plants, another 400 , including the early spider orchid, mimic females and promise their pollinators sex.

Researchers have recently dissected the package of pollen and fly to a more distant patch of flowers, reducing the chance of landing on a close relative of the original plant ${ }^{2}$. "To be deceptive means that the orchids have less sex, but the sex is better because it's not with a close relative," says Salvatore Cozzolino, an evolutionary biologist at the University of Naples in Italy.

A few years ago, Florian Schiestl, a biologist at the Swiss Federal Institute of Technol- ogy in Zurich, set out to determine just what makes orchids masquerading as females so attractive to insects. Although the flowers do look and feel a bit like a female - the hairs on O. sphegodes flowers, for example, resemble those on a female bee - Schiestl knew the key to long-distance attraction was to smell like a female, because bees respond more to distant odours than to visual cues. But plants produce hundreds of volatile compounds to repel predators and microbes, so the precise perfume was difficult to determine. "You don't know which are important for the pollinators and which are not," Schiestl says.

\section{Sexy smell}

Schiestl and his colleague Wittko Francke of the University of Hamburg in Germany reasoned that the way to intoxicate a bee must be through its antennae. Bee antennae are carpeted with hundreds of sensors that carry receptors to detect odours and dispatch electrical signals to the brain. To figure out which floral compounds were attracting male pollinators, the researchers extracted compounds from the labellum of the flower, where insects land, and then separated the chemicals using gas chromatography. They plucked a bee antenna, placed it between two electrodes and exposed it to each component of the mix. When a compound bound a receptor on the antenna, a tiny current passed through the apparatus.

Using this method, Schiestl and Francke were able to show that the complex perfume that arouses male $A$. nigroaenea bees is made up of 14 different compounds that are also common components of the waxy cuticle 
that protects the surface of many plants ${ }^{3}$. They showed that the same combination of compounds is present in the volatile sex pheromone that a female bee uses to attract a mate, and that a blend of these chemicals could make bees mate with dummy flowers. The finding also revealed how sexual deception could have evolved in this species by gradual modification of systems the plant was already using to make its own compounds. Each tweak in the ratio of compounds that increased pollinator visitation would have given the orchid a reproductive advantage.

The discovery also suggested one way in which orchids could evolve new species. Most insects are very picky about the scent they respond to and often pollinate only one orchid species. So if a plant changed its aromatic bouquet enough it would attract a different pollinator and rebuff the old one. Over time, that would - theoretically at least result in genetic isolation and the creation of a new orchid species. "We think that speciation can occur fairly quickly in that system," says Schiestl. "The plants need only to change their odour bouquet to attract a new pollinator."

The team found evidence to back this idea in the orchid blooms of Australia. They repeated the experiment on the orchid Chiloglottis trapeziformis, which tricks the male thynnine wasp (Neozeleboria cryptoides). Analysis of C. trapeziformis scent revealed a surprise - rather than adapting existing mechanisms, the orchid was producing an entirely different chemical compound they named chiloglottone, which is also a pheromone made by female wasps ${ }^{4}$. They also found that another Ophrys species, O. speculum, concocts a different wasp pheromone by developing several novel compounds ${ }^{5}$. In this case, the orchid mimic worked so well that, when offered a choice between a female or an orchid, male wasps courted the orchid.

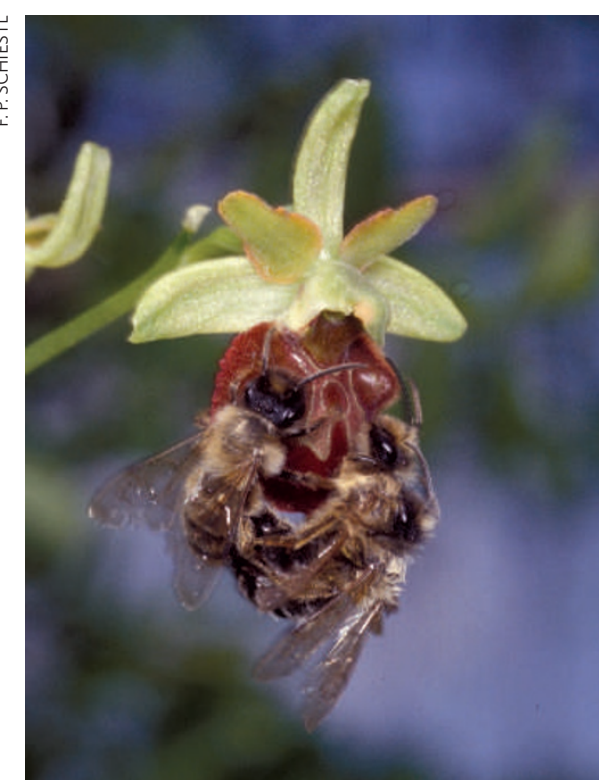

Faking it: the early spider orchid lures male bees to its pollen by mimicking the female sex pheromone.

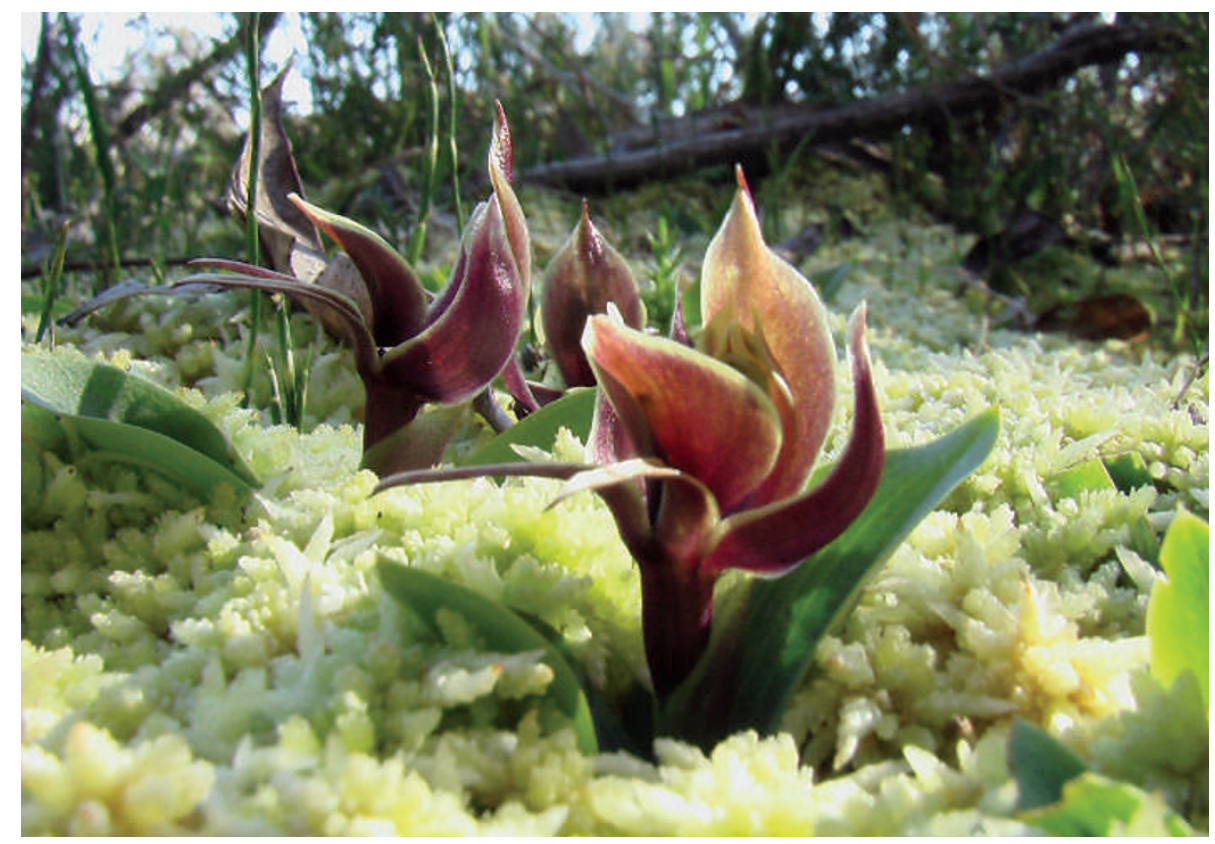

Some species of Chiloglottis orchids look identical but use different chemicals to attract pollinators.

Rod Peakall, an evolutionary biologist at Australian National University in Canberra and his colleagues have now surveyed 10 of the 30 known species of Chiloglottis orchids across Australia. In some regions, the team found orchids growing together that were identical in appearance, location and flowering time yet were, based on genetic markers, not interbreeding and that belonged to different species. Invariably, flowers of the two species produced slightly different pheromones: one, for example, produced chiloglottone alone whereas the other mixed chiloglottone and a slightly modified version. The two pheromones attract different species of wasp, and targeting different pollinators seemed to have defined species boundaries by preventing the flow of genetic information between the two sets of orchids.

\section{Growing apart}

If Peakall can prove that is the case, he may have evidence for sympatric speciation. This has been notoriously difficult to confirm because, in many systems, it is tough to prove that two similar species growing together were not spatially separated in the past and then reunited. The few documented examples have been in geographically remote areas, such as isolated islands or crater lakes, where the influx of species from other regions is unlikely. "Having solid evidence of sympatric speciation in a continental setting would be a big deal," says Daniel Bolnick, an evolutionary biologist at the University of Texas, Austin, "but it would have to be very strong evidence."

Peakall plans to seek such evidence by using small regions of highly variable genome sequences to track the evolutionary history of his orchids. The aim is to show that the two species growing together are more closely related to one another than either is to orchids located farther away. To do this, he must piece together a precise orchid family tree and rule out the possibility that two similar species actually evolved because they were once geographically separated and later came to grow side by side again. Cozzolino warns that this will be tough because the DNA sequences of these orchids do not vary by much and it may be difficult to distinguish between very closely related species. Nevertheless, Cozzolino agrees that sympatric speciation may be under way, and is looking for evidence of it among European sexually deceptive orchids as well.

And what of the insects in this web of deception? Both orchid and insect enter into the encounter in the hope of better sex - but only the flower comes away with it. This apparent inequality is such that Charles Darwin refused to believe that pollinators would waste their efforts on orchids that offer no reward. He expected the insects to figure out the ruse and save energy by avoiding the flowers and forcing the orchid into extinction.

But evolutionary biologists now know that young insects gain more by enthusiastic but indiscriminate mating than they would by being more choosy. Females are often in short supply and there is intense competition among males to find a mate - so it is better for males to try and fail than not to try at all. "The males aren't too picky," Peakall says, "Their strategy is 'Hey, I will go for anything that looks like a female because I can't afford not to." Heidi Ledford writes for Nature from Berkeley, California.

. Tremblay, R. L., Ackerman, J. D., Zimmerman, J. K \& Calvo, R. N. Biol. J. Linnean Soc. 84, 1-54 (2005).

2. Johnson, S. D., Craig, P. I. \& Ågren. J. Proc. R. Soc. Lond. B 271, 803-809 (2004)

Schiestl, F. P. et al. Nature 399, 421-422 (1999)

4. Schiestl, F. P. et al. Science 302, 437-438 (2003).

5. Ayasse, M., Schiestl, F. P., Paulus, H. F., Ibarra, F. \& Francke, W. Proc. R. Soc. Lond. B 270, 517-522 (2003). 